\title{
Polymodal Sensory Function of the Caenorhabditis elegans OCR-2 Channel Arises from Distinct Intrinsic Determinants within the Protein and Is Selectively Conserved in Mammalian TRPV Proteins
}

\author{
Irina Sokolchik, ${ }^{1}$ Takahiro Tanabe, ${ }^{1}$ Pierre F. Baldi, ${ }^{2,3,4}$ and Ji Ying Sze ${ }^{1}$ \\ ${ }^{1}$ Department of Anatomy and Neurobiology, ${ }^{2}$ School of Information and Computer Science, ${ }^{3}$ Department of Biological Chemistry, and ${ }^{4}$ Institute for \\ Genomics and Bioinformatics, University of California, Irvine, California 92697
}

Caenorhabditis elegans OCR-2 (OSM-9 and capsaicin receptor-related) is a TRPV (vanilloid subfamily of transient receptor potential channel) protein that regulates serotonin (5-HT) biosynthesis in chemosensory neurons and also mediates olfactory and osmotic sensation. Here, we identify the molecular basis for the polymodal function of OCR-2 in its native cellular environment. We show that OCR-2 function in 5-HT production and osmotic sensing is governed by its $\mathrm{N}$-terminal region upstream of the ankyrin repeats domain, but the diacetyl sensitivity is mediated by independent mechanisms. The ocr-2(yz5) mutation results in a glycine-to-glutamate substitution (G36E) within the N-terminal region. The G36E substitution causes dramatic downregulation of 5-HT synthesis in the ADF neurons, eliminates osmosensation mediated by the ASH neurons, but does not affect the response to the odorant diacetyl mediated by the AWA neurons. Conversely, wild-type sequence of the N-terminal segment confers osmotic sensitivity and upregulation of 5-HT production to a normally insensitive $C$. elegans homolog, OCR-4, but this chimeric channel does not respond to diacetyl stimuli. Furthermore, expression of either the mouse or human TRPV2 gene under the ocr-2 promoter can substantially restore 5-HT biosynthesis in ocr-2-null mutants but cannot improve the deficits in osmotic or olfactory sensation, suggesting that TRPV2 can substitute for the role of OCR-2 only in serotonergic neurons. Thus, different sensory functions of OCR-2 arise from separable intrinsic determinants, and specific functional properties of TRPV channel proteins may be selectively conserved across phyla.

Key words: TRPV channel; sensory modality; serotonin; behavior; C. elegans; gene expression

\section{Introduction}

Detecting and responding to external signals involve activation of plasma membrane receptors. Most receptors are modality specific; each receptor is activated by a unique stimulus, such as an odorant, a taste, or a hormone, to induce a specific cellular and behavioral response, such as attraction, repulsion, or growth. The specificity of the signaling is believed to form the fundamental basis of sensory discrimination and behavioral sensitivity. Remarkably, every TRPV (vanilloid subfamily of transient receptor potential channel) studied to date can be activated by multiple distinct stimuli (Gunthorpe et al., 2002; Clapham 2003). In the case of vertebrate TRPV1, which can be activated by vanilloid, heat, and acids, it is thought that the channel integrates diverse

Received July 29, 2004; revised Nov. 23, 2004; accepted Dec. 7, 2004.

This work was supported by grants from the Whitehall Foundation (J.S.), National Institutes of Health (J.S. and P.B.), and National Science Foundation (P.B.). We thank Drs. C. Bargmann for comments on this manuscript and for providing strains, F. Jurnak for critical evaluation of predicted structures, J. Gargus for inspiring discussions and suggestions during the study and providing us image clones of mouse and human TRPV2, and W. Liedtke for rat TPRV4 clone.

Correspondence should be addressed to Ji Ying Sze, Department of Anatomy and Neurobiology, University of California, Irvine, 324 Sprague Hall, Irvine, CA 92697. E-mail: jsze@uci.edu.

D01:10.1523/JNEUROSCI.3107-04.2005

Copyright $\odot 2005$ Society for Neuroscience $\quad$ 0270-6474/05/251015-09\$15.00/0 stimuli into the perception of pain, and the activity of the channel reflects combinations of the stimuli (Tominaga et al., 1998; Caterina et al., 2000). On the other hand, TRPV2 can be activated by noxious heat in heterologously expressed systems (Caterina et al., 1999) and by insulin-like growth factor (IGF)-induced subcellular translocation (Kanzaki et al., 1999), and TRPV4 senses osmotic stimuli, heat, and fatty acids (Guler et al., 2002; Watanabe et al., 2002; Alessandri-Haber et al., 2003; Liedtke and Friedman, 2003). These stimuli are likely to produce distinct perceptions and thereby to cause different responses. An intriguing question is how does a TRPV channel discriminate sensory signals in an organism exposed to the complex environment and produce specific sensory perception and behavioral responses?

TRPV proteins are a subfamily of the TRP superfamily of cation channel proteins. TRP proteins form tetrameric ion channels with each subunit containing six transmembrane segments, a $\mathrm{P}$ loop that forms a central pore, a cytoplasmic $\mathrm{N}$ terminus, and a cytoplasmic C terminus (Clapham, 2003). The $\mathrm{N}$-terminal region of TRPV proteins characteristically contains three or more ankyrin motif repeats, which are followed by the conserved transmembrane domain, but the sequence before the ankyrin repeats is less conserved among the family members (Montell et al., 2002). In some cases, sensory modalities must be governed by 
intrinsic properties of individual channel proteins because heterologously expressed channels can be activated by diverse stimuli. This notion is further supported by the identification of amino acid residues in the transmembrane domain of the TRPV1 protein necessary and sufficient to confer vanilloids sensitivity (Jordt and Julius, 2002). However, IGFs and other sensory signals appear to regulate TRPV channels indirectly (Colbert et al., 1997; Boels et al., 2001). TRPV channels are expressed broadly in both the peripheral nervous system and the CNS (Tominaga et al., 1998; Mezey et al., 2000; Delany et al., 2001). Therefore, one TRPV channel may fulfill a range of roles in detecting and responding to environmental signals. It is unclear what role TRPV channel proteins play in the selectivity of signaling transduction in the native cellular environment of an organism.

The functions of the TRPV channel encoded by the Caenorhabditis elegans ocr-2 and osm-9 genes in chemosensory neurons have been studied genetically (Colbert et al., 1997; de Bono et al., 2002; Tobin et al., 2002; Zhang et al., 2004). ocr-2 and osm-9 are coexpressed in four pairs of chemosensory neurons: the ADF serotonergic chemosensory neurons, the AWA olfactory neurons, the ASH osmotic sensory neurons, and the ADL chemosensory neurons (Tobin et al., 2002). These chemosensory neurons have ciliated sensory endings exposed to the external environment (White et al., 1986). Both OSM-9 and OCR-2 are localized to the cilia, as well as to the plasma membrane (Tobin et al., 2002). Null mutations in either ocr-2 or osm-9 cause downregulation of the expression of the tph-1 gene, which encodes the key serotonin (5-HT) biosynthetic enzyme tryptophan hydroxylase, in the ADF neurons (Zhang et al., 2004), and the odr-10 gene, which encodes a receptor for the odorant diacetyl (Sengupta et al., 1996), in the AWA neurons (Tobin et al., 2002). Both OCR-2 and OSM-9 also are required for ASH-mediated osmotic sensation and ASH- and ADL-mediated olfactory avoidance (Colbert et al., 1997; Tobin et al., 2002); however, several efforts have not been able to identify an effect of the activity of this channel on the expression of sensory components in ASH and ADL (I. Sokolchik and J. Sze, unpublished observations). OCR-2 and OSM-9 require each other for routing to the sensory cilia of the chemosensory neurons (Tobin et al., 2002), although the exact stoichiometric composition of the functional channel has not been determined. The essential function of OCR-2 in these sensory neurons provides us a tractable system in which to investigate in vivo the role of a TRPV protein in specific sensory functions.

Previous studies imply that the OCR-2/OSM-9 TRPV channel regulates different sensory functions via distinct signaling transduction pathways (Zhang et al., 2004). The odr-3 gene encodes a $\mathrm{G} \alpha$-protein and is expressed in all ocr-2-expressing amphid chemosensory neurons. ODR-3 is an essential component for the OCR-2/OSM-9 channel to mediate the olfactory and osmotic sensation (Colbert et al., 1997; Roayaie et al., 1998; Tobin et al., 2002). However, ocr-2- and osm-9-dependent transcriptional regulation of $t p h-1$ expression in the ADF neurons is unaffected by deletion mutations in $o d r-3$ or two other $\mathrm{G} \alpha$-proteins expressed in ADF (Zhang et al., 2004). Furthermore, the ASH neurons mediate osmotic sensation as well as social behavior, and both responses are dependent on OCR-2 and OSM-9; only osmotic sensation, not the social behavior, requires ODR-3 (de Bono et al., 2002). The effect of ODR-3 on olfactory and osmotic sensation indicates that some signaling components are common for different OCR-2/OSM-9 signaling pathways. However, normal 5-HT synthesis and social behavior in odr-3 mutants suggest that the OCR-2/OSM-9 channel controls these behavioral outputs by interacting with other signaling components.
Here we identify intrinsic properties of the ocr-2 protein that discern OCR-2/OSM-9 channel functions. We show that OCR-2 uses distinct structural determinants to dictate different functional modalities. We discover that the cytoplasmic N-terminal region upstream of the ankyrin repeats domain of OCR-2 specifically dictates the 5-HT synthesis and osmotic sensation. We find that both mouse and human TRPV2 can substantially activate the ADF neurons to upregulate tph-1 expression in ocr-2 deletion mutants, but neither of them improves the olfactory or osmotic sensory deficits. We propose that polymodal function of TRPV channels arises from multiple structural determinants within the channel protein, with each determinant coupled to a specific signaling transduction cascade.

\section{Materials and Methods}

Worm strains. Maintenance and manipulation of C. elegans have been described previously (Brenner, 1974). Worms were cultivated at $20^{\circ} \mathrm{C}$ and fed with Escherichia coli OP50. Wild type (WT) is Caenorhabditis elegans variety Bristol strain (N2). The following genes and alleles were used in this work: osm-9(yz6) (Zhang et al., 2004), ocr-2(yz5) (Zhang et al., 2004), ocr-2(ak47) (Tobin et al., 2002), odr-10(ky225) (Sengupta et al., 1996), ocr-2(ak47);osm-9(ky10) (Tobin et al., 2002), ocr-2(yz5);osm9(yz6) (Zhang et al., 2004), and osm-3(n1540) (Shakir et al., 1993).

Constructs and transgenic lines. All of the fusion constructs were generated by PCR. A $2.5 \mathrm{~kb}$ sequence upstream from the translational start of the ocr-2 gene was used to direct the expression of all of the transgenes, and every construct had the sequence corresponding to the FLAG epitope (Sigma, St. Louis, MO) inserted at the end of coding sequence, which was then fused to the unc-54 $3^{\prime}$-untranslated sequence in the plasmid pPD97.75 (from A. Fire, Stanford University, Stanford, CA). Coding sequences in individual transgenes are as follows: OCR-2(+), introns and exons of ocr-2 amplified from WT genomic DNA; OCR-2(G36E), introns and exons of ocr-2 amplified from ocr-2(yz5) mutants and the sequence corresponding to the RFP (red fluorescent protein) in the plasmid pPD95.75-DsRed2 (from O. Hobert, Columbia University, New York, NY); OCR-4, introns and exons of WT ocr-4 and the RPF sequence; OCR-2::OCR-4, the genomic sequence from the translational start to codon 186 of ocr-2 fused to the ocr- 4 genomic sequence spanning codons 145 to the end of the coding region; mTRPV2, the cDNA sequence of mouse TRPV2 in a American Type Culture Collection (Manassas, VA) clone, IMAGE ID 3487527; hTRPV2, the cDNA sequence from human TRPV2 in the American Type Culture Collection clone, IMAGE ID 4298484; and rTRPV4, the cDNA sequence from rat TRPV4 (from W. Liedtke, Duke University, Durham, NC).

For each construct, products from at least two independent PCR reactions were pooled to reduce potential errors from PCR. Purified PCR products $(70 \mathrm{ng} / \mu \mathrm{l})$ were microinjected into ocr-2(ak47) mutant worms carrying an integrated tph-1::gfp reporter (Sze et al., 2000). Therefore, the same GFP (green fluorescent protein) reporter was observed in different genetic backgrounds. A plasmid containing elt-2::gfp (a gift from J. McGhee, University of Calgary, Calgary, Canada) was coinjected as a transgenic marker. Except for hTRPV2, which has one transgenic line, two to four transgenic lines from each construct in at least three generations were examined. To test the effect of the transgenes on sensory behaviors, the tph-1::gfp reporter was outcrossed from the corresponding transgenic lines, i.e., the same TRPV transgenes were tested in different neuronal functions.

GFP expression and immunoanalysis. Animals from various developmental stages were examined using a Zeiss (Oberkochen, Germany) Axio plan II equipped with a fluorescence light source. The expression pattern of the integrated $t p h-1:: g f p$ transgene in WT, ocr-2, and osm-9 mutant animals has been published previously (Sze et al., 2002; Zhang et al., 2004).

To quantify GFP intensity of tph-1::gfp expression in the ADF neurons, the images were captured with a Zeiss AxioCam digital camera at a fixed exposure time, and the fluorescence within a $25 \times 25$ pixel area of the cell body was scored. Hence, the same dimension of the ADF neurons in different genetic background was compared. 


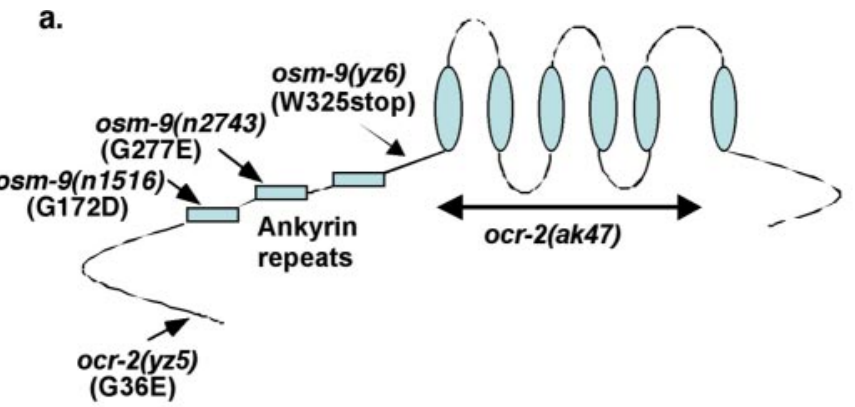

\section{c. anti-FLAG antibody staining}

\section{b. anti- $5 \mathrm{HT}$ antibody staining}
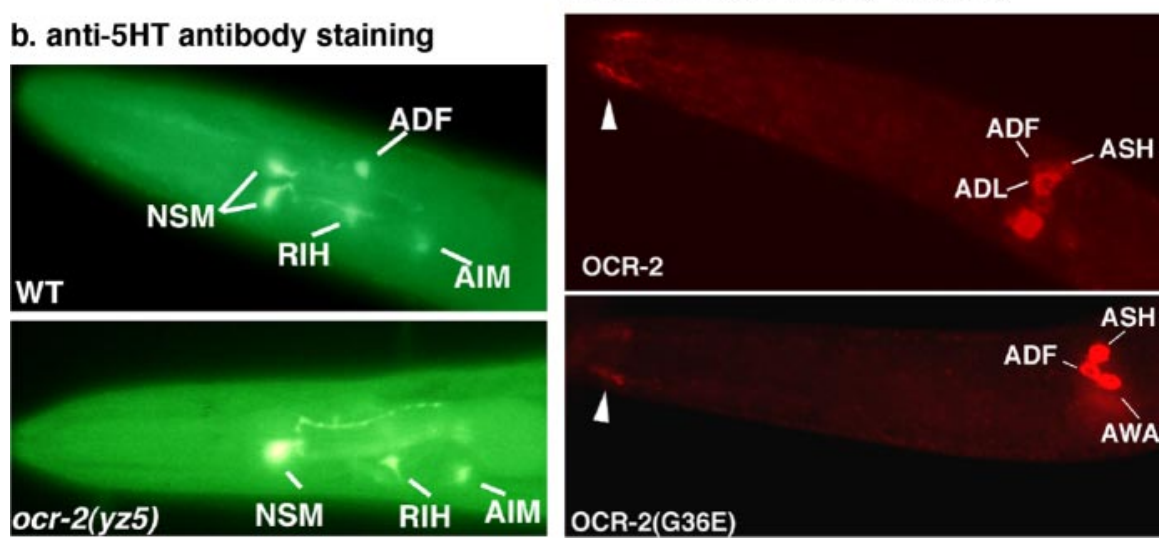

Figure 1. The ocr-2(yz5) G36E substitution disrupts $O C R-2$ function in the serotonergic ADF neurons. $a$, Schematic representation of ocr-2 and osm- 9 alleles used in this study. The ocr- 2 and osm- 9 proteins share the same domain structure and topology (Harteneck et al., 2000; Tobin et al., 2002). The ocr-2(ak47) and osm-9(yz6) mutations result in channel proteins lacking the transmembrane domain (indicated as cylinders) and are expected to be functional null alleles. It is noteworthy that the osm9 (n2743) and osm-9(n1516) mutations each result in a amino acid substitution in the ankyrin motifs and both cause deficits in every 0SM-9/0CR-2 function (Colbert et al., 1997; Zhang et al., 2004), whereas the ocr-2(yz5) mutation discriminatively affects the functions. The position of amino acid changes corresponds, respectively, to the OSM-9 and OCR-2 protein sequences. $b$, Analysis of 5-HT immunoreactivity in WT and ocr-2(yz5) mutant animals. In WT, seven neurons of four classes in the head and two HSN neurons around the vulva (data not shown) are stained by the anti-5-HT antibody. In ocr-2(yz5) mutants, 5-HT immunoreactivity in the ADF neurons is dramatically reduced or absent, but 5-HT immunoreactivity in the other neurons is unaffected. Both ocr-2 and osm- 9 are expressed in ADF but not in the other serotonergic neurons (Tobin et al., 2002; Zhang et al., 2004). Adult animals are shown, and the anterior is toward the left. C, Analysis of the expression pattern of FLAG-tagged OCR-2 and OCR2(G36E). The FLAG epitope was tagged at the C terminus in both constructs. Consistent with the expression pattern revealed by ocr-2::gfp (Tobin et al., 2002), the immunofluorescence is predominantly observed in the cilia and plasma membrane of the ADF, ASH, ADL, and AWA neurons in the head and the PHA and PHB neurons in the tail; representative photomicrographs are shown. In general, the staining in the cilia is much weaker than in the plasma membrane, even with the OCR-2 construct. FLAG immunoreactivity of the $0 C R-2(G 36 \mathrm{E})$ fusion construct is lower overall, with a stronger effect observable in the cilia.

The staining of anti-5-HT antibody was performed using the McIntire-Horvitz whole-mount procedure (McIntire et al., 1992), with modifications (Sze et al., 2000). To conduct anti-FLAG antibody staining, animals were fixed according to an established protocol (Finney and Ruvkun, 1990) and stained with monoclonal anti-FLAG antibody M2 (1:200; Sigma), and the staining pattern was detected using Alexa Fluor 568 rabbit anti-mouse antibody (Molecular Probes, Eugene, OR) at the dilution 1:2000.

Behavioral assays. Behavioral assays were conducted with well fed young adult animals. Odor attraction assays were performed as described by Bargmann et al. (1993). Unless specified, the dilutions of odorants in ethanol were as follows: 1:200 benzaldehyde, 1:200 isoamyl alcohol, and 1:1000 diacetyl. The odortaxis index was calculated as [(number of animals at attractant) - (number of animals at solvent ethanol)]/(total number of animals moved).

Osmotic avoidance was assayed as described by Vowels and Thomas (1994). Briefly, 10-15 young adult animals from a strain were transferred to an agar plate without food and allowed to migrate briefly to remove excess bacteria. These animals were then placed on a fresh agar plate in the center surrounded by a ring $(16 \mathrm{~mm}$ in diameter) of high-osmotic-strength solution (7 M glycerol). Animals that crossed the ring within 15 min were scored as being osmotic insensitive. The osmotic sensitivity was calculated as the ratio of the number of worms remaining within the ring divided by the total number of animals assayed.

Sequence and structural analysis. Multiple alignments of TRPV channel protein sequences were produced using ClustalW (Higgins et al., 1994), HMMpro (Baldi et al., 1994), and T-coffee (Notredame et al., 2000) algorithms. Structural features and three-dimensional structure were predicted using the SCRATCH suite of predictors (http://www.igb.uci.edu/ servers/psss.html). Secondary structure was predicted using the current version of the SSpro program (Baldi et al., 1999; Pollastri et al., 2002b). Relative solvent accessibility was predicted using the current version of the ACCpro program (Pollastri et al., 2002a). On large validation data sets, SSpro and ACCpro have been shown to achieve correct prediction rates in the $75-80 \%$ range at the level of single amino acids. Tertiary structures were derived from predicted contact maps using the methods described by Baldi and Pollastri (2003). Structural predictions were also derived using the PSIPRED server (McGuffin et al., 2000).

\section{Results}

The ocr-2(yz5) glycine-to-glutamate

(G36E) substitution results in a

complete loss of OCR-2/OSM-9 channel

function in the serotonergic

ADF neurons

The serotonergic phenotype can be precisely identified within the nervous system of C. elegans. Of the 302 neurons present in an adult C. elegans hermaphroditic worm, nine neurons composed of five neuronal classes are stained by antibody raised against 5-HT (Horvitz et al., 1982; Sze et al., 2000) (Fig. 1). The pair of ADF neurons is the only serotonergic sensory neurons. The ADF neurons reside in the bilaterally symmetric amphids and have their ciliated sensory endings exposed to the external environment (White et al., 1986). The ADF neurons have been proposed to mediate neuroendocrine response to the environment. Worms with the ADF neurons ablated by a laser beam (Bargmann et al., 1991b) or bearing a mutation that abrogates ADF 5-HT biosynthesis (Zhang et al., 2004) are more susceptible to developmental and metabolic arrest.

Through genetic analysis of serotonergic phenotypes in $C$. elegans, we identified a set of genes that specifically regulate 5-HT biosynthesis in the ADF neurons (Sze et al., 2002). Two of the genes, osm-9 and ocr-2, regulate the expression of the tryptophan hydroxylase gene $t p h-1$, which is essential for 5-HT biosynthesis (Zhang et al., 2004). We have shown that deletion mutations in either osm-9 or ocr-2 dramatically downregulate the expression of a green fluorescent protein reporter of tph-1 (tph-1::gfp) and diminish/abolish 5-HT immunoreactivity in the ADF neurons, but the morphology and survival of the neurons are unaffected (Zhang et al., 2004). Misexpression of osm-9 and ocr-2 under 
neuron-specific promoters revealed that OSM-9 and OCR-2 act cell autonomously to regulate tph-1 expression (Zhang et al., 2004). ocr-2 and osm-9 mutations do not appear to cause a general reduction in gene expression. We examined five ADF neuronal marker genes: $t p h-1$ expression is eliminated, but the others are unaffected (Zhang et al., 2004). Therefore, a signaling pathway from the OSM-9/OCR-2 TRPV channel transduces events detected at the ADF cell surface into a specific transcriptional regulatory program, 5-HT synthesis, and 5-HT downstream signaling.

The ocr-2(yz5) allele was identified previously from a genetic screen for mutations that downregulate $t p h-1:: g f p$ expression in the ADF neurons (Sze et al., 2002). The ocr-2(yz5) mutation is a single nucleotide change that results in a glycine-to-glutamate (G36E) substitution (Fig. 1a). Staining of anti-5-HT antibody indicates that ADF 5-HT immunoreactivity is nearly eliminated in ocr-2(yz5) mutants (Zhang et al., 2004) (Fig. 1b). The levels of tph-1::gfp expression and 5-HT immunoreactivity in ocr-2(yz5) mutants are indistinguishable from that seen in mutants bearing the ocr-2(ak47) deletion allele, which removes the first five transmembrane segments, or in mutants bearing a double mutation of both ocr-2(yz5) and osm-9 deletion (Zhang et al., 2004). Together, these results suggest that the G36E substitution results in a severe or complete loss of OCR-2/OSM-9 channel function in the ADF neurons.

There are two possible mechanisms through which such a strong phenotype could be produced by a single amino acid substitution perturbing a critical site: either the protein cannot be expressed or cannot be activated. To test the first possibility, we compared the expression pattern of the OCR-2(G36E) protein with OCR-2 (Fig. 1c). We tagged a FLAG epitope to the $\mathrm{C}$ terminus of OCR-2 and OCR-2(G36E), introduced the fusion constructs individually into ocr-2(ak47) mutants, and stained the transgenic lines with anti-FLAG antibody. Consistent with the expression pattern revealed by ocr-2::gfp (Tobin et al., 2002), the immunoreactivity of OCR-2::FLAG and OCR-2(G36E)::FLAG is detected in the cilia and plasma membrane of the chemosensory neurons ADF, AWA, ASH, and ADL (Fig. 1c). However, whereas ocr-2(ak47) animals carrying the OCR-2::FLAG transgene restore tph-1 expression (68\% show strong tph-1::gfp in ADF; $n=79)$, ocr-2(ak47) animals that carry OCR-2(G36E)::FLAG $(n=79)$ exhibit reduced tph-1::gfp expression as their nontransgenic siblings $(n=235)$. These data indicate that FLAG-tagged OCR-2 is localized to the cellular sites in which OCR-2 acts and that the G36E substitution does not alter the subcellular localization. These results suggest that the G36E substitution disrupts the function of the OCR-2/OSM-9 channel in the ADF neurons.

The immunofluorescence of OCR-2(G36E)::FLAG is relatively lower than that of OCR-2::FLAG (Fig. 1c). This could reflect the mosaicism of the transgenic arrays (Mello et al., 1991). Alternatively, it could be an indication of decreased stability of OCR-2(G36E) resulting from altered structure and/or from its inability to bind to OCR-2 partners.

\section{Neuron-specific effect of the OCR-2(G36E) substitution}

In addition to regulate tph-1 expression in the ADF neurons, OCR-2 acts in the ASH and AWA neurons to mediate a range of sensory behaviors (Tobin et al., 2002). The products of the deletion allele ocr-2(ak47) cannot form a channel, and ocr-2(ak47) mutant animals are defective in each of the functions mediated by these neurons (de Bono et al., 2002; Tobin et al., 2002; Zhang et al., 2004). We asked whether the G36E substitution results in a complete inactivation of the channel and thereby disrupts every

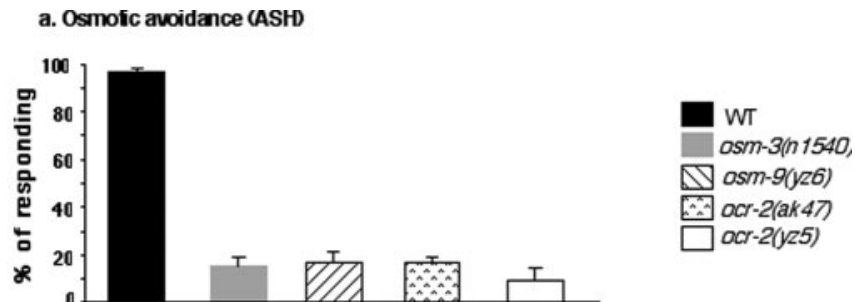

b. Olfactory attraction
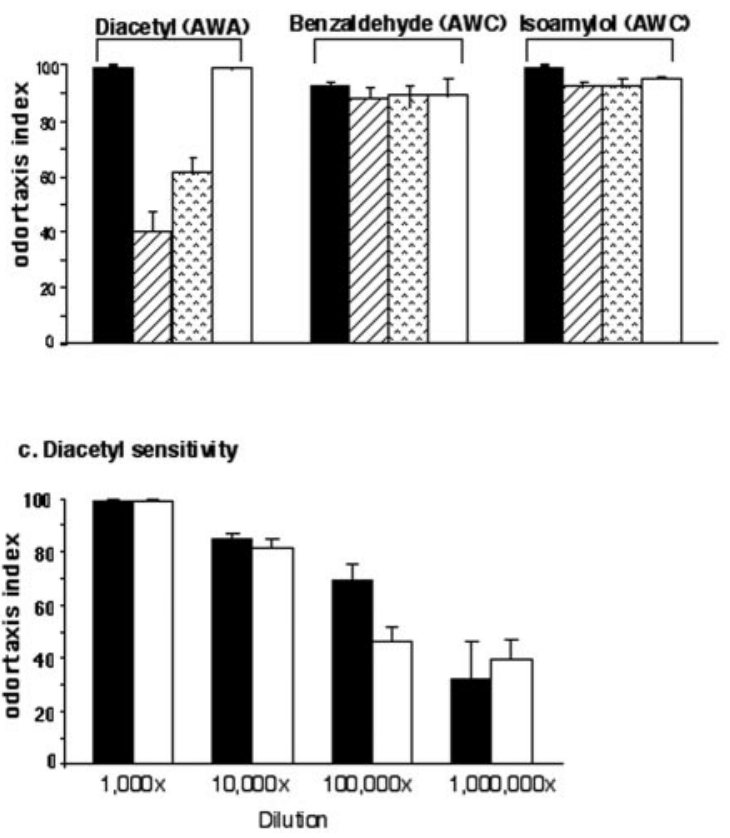

Figure 2. The ocr-2(yz5) G36E substitution selectively abrogates 0 CR-2 sensory functions. $a$, ocr-2(yz5) mutant animals fail to respond to osmotic stimuli. The assay tests for avoidance of 7 M glycerol. WT animals are repelled by high-osmolarity solutions and cannot escape from the ring of $7 \mathrm{~m}$ glycerol. osm-3 mutants have compromised sensory endings and presumably cannot sense; $\sim 85 \%$ of osm-3(n1540) mutants crossed the ring (Shakir et al., 1993). We repeatedly observed that higher percentages of ocr-2(yz5) mutants cross the ring than ocr-2(ak47), osm9 (yz6), or osm-3 mutants. b, ocr-2(yz5) mutants respond as well as WT to the odor attractant diacetyl. ocr-2(ak47) and osm-9(yz6) mutants exhibit profound deficits in sensing diacetyl at the dilution 1:1000. All of the mutants respond normally to benzaldehyde and isoamyl alcohol mediated by the AWC neurons. OCR-2 is not expressed in AWC, but it is expressed in AWA, which mediates diacetyl sensation. c, Comparison of diacetyl sensitivity between WT and ocr-2(yz5) mutant animals in series dilution assays. The neurons sensing the odorants are indicated. Each bar represents the mean \pm SEM of at least three assays.

function of the channel or whether it alters a structural determinant that is specific for its action in the serotonergic signaling pathway. To evaluate the effect of the OCR-2(G36E) substitution in ASH and AWA function, we compared ASH-mediated osmotic sensation and AWA-mediated odor attractant diacetyl sensation in ocr-2(yz5) mutants with that of ocr-2(ak47) or osm9(yz6) deletion mutants (Fig. 2).

When touching a high osmotic solution, C. elegans evokes a backward movement (Culotti and Russell, 1978). This osmotic sensation is mediated by the ASH chemosensory neurons (Bargmann et al., 1990; Kaplan and Horvitz, 1993) and requires both OCR-2 and OSM-9 (Colbert et al., 1997; Tobin et al., 2002). The response to osmotic stimuli is almost completely abolished in ocr-2(yz5) mutants as in ocr-2(ak47) and osm-9(yz6) deletion mutants (Fig. 2a). Thus, the G36E substitution also abolishes the channel function in the osmotic sensation mediated by the ASH neurons, confirming the importance of the G36 residue. 
a. Predicted 3D structure (aa 1-160)

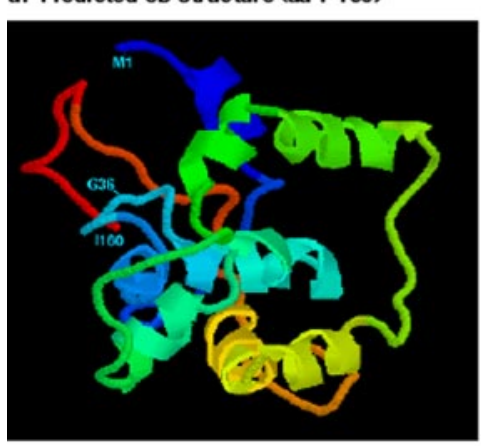

b. Secondary structure and relative solvent accessibility oct-2WT

MGSSSSTQSSTRATFVGIGRLREDIIKTYQLTDTHGGGELLPTLRYAIISSDT SFID

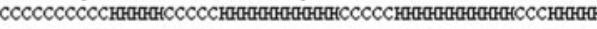
eеeеeеeеe-ee-----e-ee--e-------eee-e-----------eee--AYLETKVKDFLYMGGKGKLVTVTELVKLRKKERNERLGAF SRKKKGKGKSGPNVLD

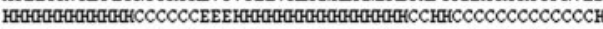

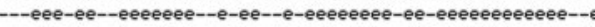

DFMOEGENTGDLKKALKLLDGGGKGGRYESKYREI SWKLEERGSMGETII FHFHसH

\section{oct-2(yz5)}

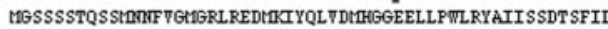

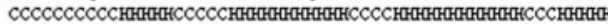

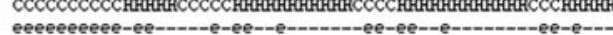
AYLETKTKDFL YMGGKGKL TTVTEL TKLRMKERTERL GAF SRKXGKGKSGPYVLD

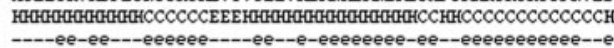
DFMOEGERTGDLKKALKLLDGGGKGGRIESKYREI STKLEERGSMGETII DFRQEGERTGDLKKKALKLLDGGGKGGRWE SKYREISWKLEERGSMGETII

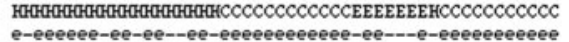

Figure 3. Structural model of the 0 CR-2 N-terminal segment. $a$, Prediction of the tertiary structure for 0 CR-2 (amino acids 1-160) (see Materials and Methods). G36 is predicted to reside within a fairly exposed coil between two $\alpha$-helices. aa, Amino acids. $b$, Prediction of the secondary structure and relative solvent accessibility for wild-type OCR-2 and OCR-2(G36E) (amino acids 1-160) (see Materials and Methods). In wild-type OCR-2, G36 resides right before an $\alpha$-helix. When $G$ is changed to $E$, the $E$ is predicted to become part of the helix. This shift could cause a global change in the side-chain phasing along the helix and alter intermolecular and intramolecular interactions. Notice the changes in the solvent accessibility of amino acids 33-60. The exposure of the residues was computed with a $30 \%$ threshold. The amino acid 36 is marked by asterisks. $\mathrm{H}, \mathrm{Helix}$; $\mathrm{C}$, coil; $\mathrm{e}$, exposed; - , buried.

In contrast, ocr-2(yz5) mutants respond as well as WT animals to the odor attractant diacetyl sensed by the AWA neurons, whereas the diacetyl sensation is significantly compromised in ocr-2(ak47) and osm-9(yz6) mutants (Fig. 2b). To determine whether the OCR-2(G36E) substitution causes a subtle effect in the sensitivity to diacetyl, we assayed the response to diacetyl in a series of dilutions. There is no substantial difference between WT and ocr-2(yz5) worms observed in response to diacetyl over 1,000,000-fold dilutions (Fig. $2 c$ ). The normal diacetyl response is consistent with proper expression and localization of the OCR$2(\mathrm{G} 36 \mathrm{E})$ protein (Fig. 1c). Thus, the G36E substitution in the ocr-2(yz5) allele prevents the channel to upregulate $t p h-1$ expression, disrupts osmotic sensation, but does not affect olfactory sensory transduction stimulated by diacetyl. The modality selective deficits of ocr-2(yz5) mutants suggest that distinct sensory functions of the TRPV channel are governed by distinct intrinsic properties within the channel protein.

Osmotic sensation and upregulation of 5-HT are governed by the $\mathrm{N}$-terminal segment upstream of the ankyrin repeats domain of OCR-2

The distinct, neuron-specific deficits displayed by ocr-2(yz5) mutants provide us with a unique opportunity to further explore the structural determinants of TRP channel proteins in modality discrimination. The N-terminal cytoplasmic region of TRPV channels is composed of two subregions (Fig. 1a): the ankyrin repeats domain adjacent to the transmembrane domain is the hallmark of TRPV proteins, and the segment upstream of the ankyrin domain exhibits less conserved sequence among the family members (supplemental Fig. 1, available at www.jneurosci.org as supplemental material). Ankyrin repeats have been shown to tether membrane proteins to the cytoskeleton and to interact with signaling molecules such as the inositol triphosphate receptor and the $\mathrm{Na}^{+} / \mathrm{H}^{+}$exchanger (Raucher et al., 2000; Denker and Barber, 2002). The ankyrin repeats are likely to be essential for TRPV channel function. A splicing variant of rat TRPV1 lacking the most part of the N-terminal region is expressed properly but

cannot be activated by any identified stimulus (Schumacher et al., 2000). Furthermore, two osm-9 alleles have two different amino acid substitutions in the ankyrin motifs (Fig. 1a); both alleles affect every function of OSM-9 (Colbert et al., 1997; Zhang et al., 2004). However, the OCR2 (G36E) substitution is located $\sim 220$ amino acids upstream from the first ankyrin motif and affects a subset of OCR-2 functions (Figs. 1, 2). Analysis of the protein sequence does not give us any strong indications of known functional domains within the segment upstream of the ankyrin repeats. To gain insights into how the G36E substitution might affect channel function, we modeled the structural features of the segment upstream of the ankyrin domain of OCR-2 (Fig. 3). Secondary structure and relative solvent accessibility prediction programs suggest that this segment can fold into a helicesrich moiety with the G36 residue located in a fairly exposed coil region between two helices (Fig. 3b). G36E substitution is predicted to shift the start of the C-terminal helical fold, alter the phasing of the side chains of the helix, and change the structure in the region (Fig. $3 b$ ).

Does this $\mathrm{N}$-terminal helical moiety contain determinants dictating OCR-2 function? If this were the case, we might expect this segment to confer OCR-2 function when it is transferred into a normally insensitive homolog. The C. elegans TRPV gene ocr-4 is expressed in neurons but does not overlap with OCR-2 (Tobin et al., 2002). Despite differences in protein sequence at the $\mathrm{N}$-terminal segment between OCR-2 and OCR-4, the secondary structure and other structural features of the OCR-4 sequence are predicted to be very similar to that of OCR-2. We first tested whether OCR-4 can substitute for OCR-2 function. We expressed the ocr-4 coding sequence under the control of the ocr-2 promoter. The FLAG epitope was inserted at the $\mathrm{C}$ terminus of the coding region for monitoring the expression. This construct was introduced into ocr-2(ak47) deletion mutants. Staining of the transgenic animals with anti-FLAG antibody shows that the OCR-4::FLAG protein is expressed and properly localized in the OCR-2-expressing cells (Fig. 4a). However, this ocr-4 transgene cannot recover tph-1::gfp expression in $\mathrm{ADF}$ (Fig. $4 b, c$ ), nor does it rescue osmotic or diacetyl sensation of the mutants (Fig. $4 d, e$ ). Because a FLAG-tagged OCR-2::FLAG transgene can efficiently rescue these phenotypes in ocr-2(ak47) (Figs. 4c,e, 5d), we conclude that the OCR-4 and OCR-2 proteins are not functionally exchangeable.

Having observed OCR-4::FLAG properly localized in OCR-2expressing cells, we generated a chimeric channel protein by substituting the OCR-2 N-terminal segment for the cognate segment in OCR-4. This chimeric construct was tagged with the FLAG epitope and expressed under the control of the ocr-2 promoter. When the OCR-2::OCR-4::FLAG construct was injected into ocr2(ak47) mutants, we find that the transgenic animals show a substantially increased $t p h-1:: g f p$ expression in the ADF neurons with respect to their nontransgenic siblings (Fig. 4b,c) and are able to respond to osmotic stimuli (Fig. 4d). However, the OCR-2::OCR-4 chimeric channel protein fails to rescue diacetyl sensory deficits of ocr-2(ak47) mutants (Fig. 4e). These results 
demonstrate that the $\mathrm{N}$-terminal segment of OCR-2 is sufficient to confer the channel function in regulation of 5-HT synthesis and osmotic sensation, but that diacetyl sensation is governed by separated determinants. Thus, a plausible explanation for the normal diacetyl response observed in ocr-2(yz5) mutants is that the G36E substitution does not affect the determinants of diacetyl sensitivity.

The G36 residue appears to be located in a region highly conserved among the ocr genes (Tobin et al., 2002) (supplemental Fig. 1, available at www.jneurosci.org as supplemental material), but OCR-4 is unable to substitute for OCR-2 function (Fig. 4 ). Hence, the glycine residue per se may not be the determinant. It remains possible that the G36E substitution may alter the structural organization of this region and change the affinity and specificity of the channel with the transduction complexes that regulate 5 -HT production and osmotic sensation.

\section{Mammalian TRPV2 genes can substitute for OCR-2 to upregulate tph-1 expression}

Previous genetic analysis implies that OCR-2/OSM-9 channel is a component in the DAF-2 (diaminofluorescein-2)/insulin receptor endocrine pathway that modulates larval development and metabolism in response to stressful environmental signals such as starvation, heat, and pheromone (Zhang et al., 2004). It has been reported that mouse TRPV2 is regulated by insulin-like growth factors, which stimulate TRPV2 translocation from intracellular compartments to the plasma membrane in the transfected cells (Kanzaki et al., 1999). Study of rat TRPV2 revealed that the channel can be activated by noxious heat $(\mathrm{Ca}-$ terina et al., 1999). TRPV2 is expressed in sensory neurons as well as broadly in the CNS (Caterina et al., 1999), but its in vivo function has not yet been reported. We speculated that these functions of OCR-2 and mammalian TRPV2 could reflect a unified, phylogenetically conserved signaling pathway that integrates the information from sensory and endocrine inputs and elicits selfpreservation responses. Having identified that OCR-2 function is determined by its intrinsic properties, we asked whether the OCR-2 shares functional properties in common with TRPV2. To address this question, we expressed the human and mouse TRPV2 cDNA (hTRPV2 and mTRPV2, respectively), each under the control of the ocr-2 promoter, and determined their ability to rescue tph-1 expression and sensory functions in ocr-2(ak47) mutants.

The FLAG-tagged hTRPV2 and mTRPV2 proteins are localized to the cilia and the plasma membrane of the chemosensory neurons (Fig. 5a), similar to OCR-2 (Fig. 1c), indicating that the mammalian TRPV2 proteins can be properly localized to the sites in which OCR-2 acts. ocr-2 mutants expressing either the mTRPV2 or hTRPV2 construct exhibit substantially increased tph-1::gfp expression in ADF than their nontransgenic siblings (Fig. $5 b, c)$. These results suggest that both hTRPV2 and mTRPV2
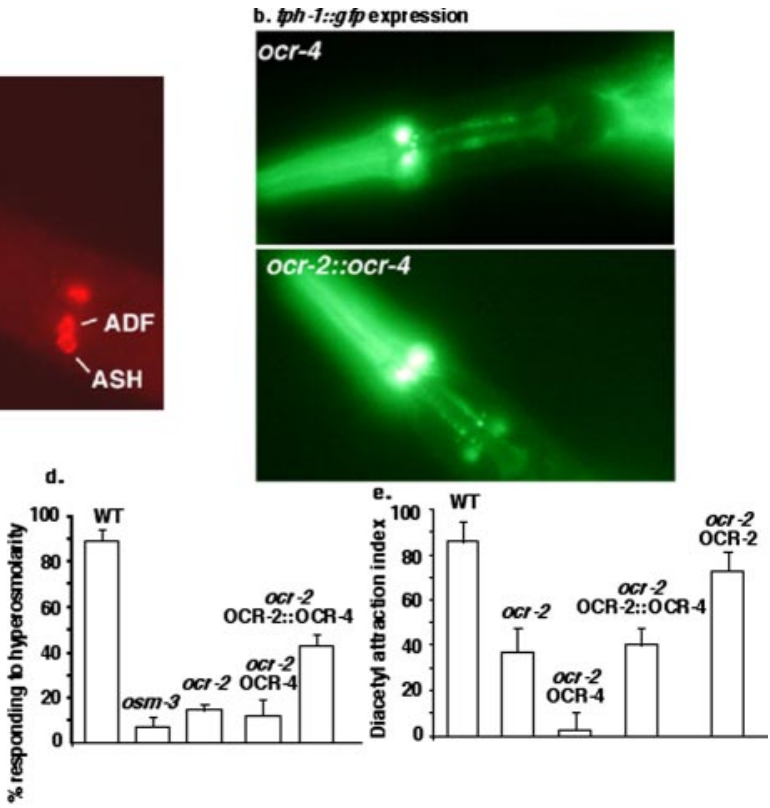

Figure 4. The $0 C R-2 \mathrm{~N}$-terminal region confers the regulation of tph-1 expression and osmotic sensation to normally insensigexpression in the ADF neurons. All of the 列 transgene exhibited severer diacetyl sensory deficits than their nontransgenic sibling, perhaps attributable to an antimorph effect of OCR-4 in AWA function. Each bar represents the mean \pm SEM of at least three assays.

can substitute for OCR-2 in the serotonergic neurons. ADF GFP in ocr-2(ak47) animals bearing TRPV2 constructs is not as strong as that seen in animals bearing an OCR-2 transgene (Figs. $4 c, 5 b$ ). Thus, TRPV2 and OCR-2 are not completely equivalent.

Upregulation of $t p h-1$ expression in the ADF neurons requires both OCR-2 and OSM-9 (Zhang et al., 2004). We tested whether the TRPV2 proteins can substitute also for OSM-9 or for both OCR-2 and OSM-9. We crossed the mTRPV2 and hTRPV2 transgenes individually into osm-9(yz6) deletion mutants and ocr-2(ak47);osm-9(ky10) double-deletion mutants and scored $t p h-1:: g f p$ expression. In each of the strains, only a very small fraction of the transgenic animals expressed tph-1::gfp slightly above the background in the ADF neurons (data not shown). These results suggest that TRPV2 and OCR-2 share the same requirement for OSM-9 to activate tph-1 expression. Unlike OCR-2, which requires OSM-9 for routing to the cilia of the chemosensory (Tobin et al., 2002), TRPV2 can be localized to the cilia in osm-9 or ocr-2;osm-9 mutants (data not shown). Because TRPV2 on its own cannot activate tph-1::gfp expression, TRPV2 apparently functions together with OSM-9 and regulates OCR2/OSM-9 signaling cascades.

We also examined the response to osmotic and diacetyl stimuli in ocr-2(ak47) transgenic animals bearing individual TRPV2 constructs. No improvement can be detected in their response to diacetyl or high-osmolarity solutions (Fig. 5d,e). Because the OCR-2::OCR-4 chimeric protein exhibits an equivalent rescue 

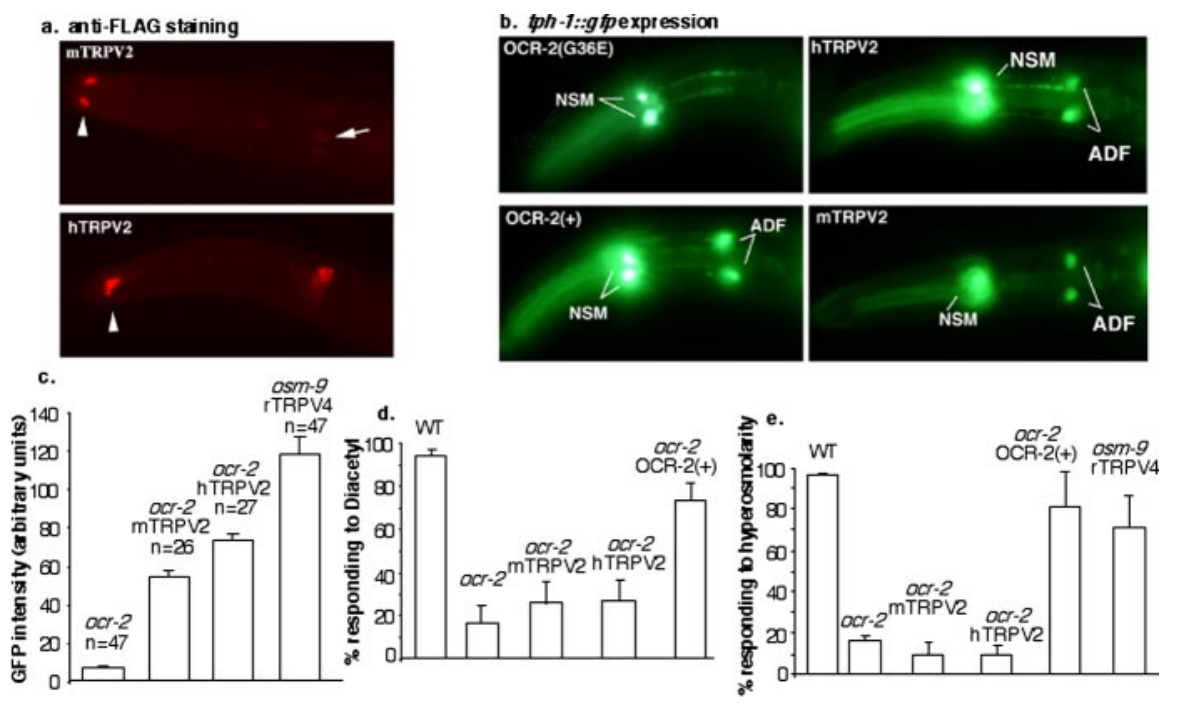

Figure 5. Mouse and human TRPV2 genes recover tph-1::gfp expression in ocr-2(ak47) mutants. a, Anti-FLAG antibody staining. FLAG-tagged mouse and human TRPV2 are localized to the cilia and plasma membrane of OCR-2-expressing chemosensory neurons. The arrowheads point to the cilia. Although the mammalian TRPV2s and OCR-2 display a very similar expression pattern and subcellular location, the TRPV2 proteins tend to be concentrated in the cilia, whereas OCR-2 is more concentrated in the cytoplasma membrane (see Fig. 1c). b, tph-1::gfp expression in ocr-2 mutants transformed with various TRPV transgenes. The transgene in each strain is indicated. ocr-2 mutants bearing the 0 CR-2(G36E) transgene show absence of tph-1::gfp expression in the ADF neurons. Mouse and human TRPV2 substantially recover tph-1::gfp expression in ADF. The GFP levels in animals transformed with the mouse and human TRPV2 is not as high as those transformed with the WT OCR-2 transgene. c, Quantification of tph-1::gfp expression in the ADF neurons. All of the strains carry the same tph-1::gfp transgene. The fluorescence in a $25 \times 25$ pixel area in the ADF neurons was quantified by measuring the pixel intensity of the images captured using an Axiocam MR digital camera, as described in Materials and Methods. GFP intensity in multiple generations of the strains was examined. $n$, Number of animals examined. $d, e$, Mouse and human TRPV2 cannot improve osmotic or diacetyl deficits in ocr-2(ak47) mutants. In contrast, the 0 CR-2 construct can rescue both osmotic and diacetyl defects. All of the transgenes are expressed under the control of the same ocr-2 promoter, and each has the FLAG epitope tagged at the C terminus of the coding region. For behavioral assays, the data represent the response of transgenic animals identified based on their transgenic marker. Each bar represents the mean \pm SEM of at least three assays.

ability in tph-1::gfp expression and osmotic sensation (Fig. $4 c, d$ ), the difference in $t p h-1$ expression and sensory function likely reflects the qualitative rather than quantitative difference of TRPV2 activity in these signaling pathways.

It has been reported that the rat TRPV4 (rTPRV4) channel protein can substitute for OSM-9 function in osmotic sensation mediated by the ASH neurons (Liedtke et al., 2003). We tested whether rTRPV4 can also substitute OSM-9 for activation $t p h-1$ expression in the ADF neurons. Supporting the published results, rTPRV4 expressed under the ocr-2 promoter nearly fully rescues osmotic sensation in the osm-9(yz6) mutant background (Fig. $5 e$ ). However, rTRPV4 only partially recovers $t p h-1:: g f p$ expression in the ADF neurons of the mutants (Fig. $5 c$ ). Together, these observations suggest that the molecular mechanisms underlying TRP channel function in 5-HT synthesis and osmotic sensation also are distinct. This result is consistent with our previous studies indicating that different signaling components are required for OCR-2/OSM-9 function in these two classes of neurons (Zhang et al., 2004). rTRPV4 osmotic sensitivity in C. elegans is enhanced when they are assayed at mammalian physiological temperatures (Liedtke et al., 2003). Because mammalian physiological temperatures are lethal to $C$. elegans, it is not known whether the activity of TRPV2s also is optimized for their native cellular temperatures, and a full activation of TRPV2 and TRPV4 can further enhance tph-1 expression.

\section{Discussion}

The results from this study reveal that the codes for the polymodal function of OCR-2 reside within this channel protein. Our work has identified a subregion within the cytoplasmic $\mathrm{N}$ terminus of OCR-2 that determines the osmotic sensitivity and confers the ability to upregulate $t p h-1$ expression in C. elegans. The ocr-2(yz5) G36E substitution within this segment results in a complete loss of these functions. The WT sequence of this subregion is sufficient to confer these functions to a normally insensitive homolog, OCR-4, demonstrating that this segment can exert the functions via a non-cognate channel. Furthermore, we find that both the mouse and human TRPV2 channel proteins can substitute for OCR-2 to upregulate $t p h-1$ expression but cannot mediate osmotic sensation, suggesting that specialized properties of TRPV proteins are selectively conserved across phyla. Our results shed light on the molecular mechanisms that allow a single channel entity to selectively respond to diverse stimuli in a defined cellular environment within an organism.

Given that many vertebrate TRPV channels can be activated by multiple stimuli in various heterologous systems, it should not be surprising that polymodal sensitivities are determined by intrinsic properties of the channel proteins. Analyses of mammalian TRPV channels have identified several sensory-specific determinants within the transmembrane regions (Jordt and Julius, 2002; Vriens et al., 2004). These functional determinants may directly influence the opening of the channels. Less expected, however, is the region upstream of the ankyrin repeats domain that determines OCR-2-specific functions. The amino acid sequence of this region is quite divergent among TRPV family members, and its role in TRPV channel signaling has not been characterized previously. Because the OCR-2(G36E) protein appears to be properly localized and is able to mediate a normal diacetyl sensation, these observations argue against the role of this $\mathrm{N}$-terminal segment in OCR-2 trafficking. Because the WT sequence of this segment confers OCR-2 functions to OCR-4, the codes for these functions must reside within this segment.

One plausible function of the cytoplasmic N-terminal segment is allosteric coupling of ligand binding and channel opening. In this scenario, the N-terminal segment folds into a helixrich moiety and blocks the channel. Binding of the ligand or receptor-coupled second messengers to this region induces allosteric alterations, which in turn cause the channel to open. The G36E substitution could prevent ligand binding or abrogate allosteric movements. This mechanism resembles the "ball-andchain" gating used by the Shaker $\mathrm{K}^{+}$channels, in which the $\mathrm{N}$-terminal residues act as the "ball" for channel inactivation (Yellen, 2002). However, it is unlikely that the G36E substitution simply causes a complete blockage of the OCR-2/OSM-9 channel, because the diacetyl sensitivity is unaffected in ocr-2(yz5) mutants. Furthermore, deletion of the first 36 amino acids or internal truncations within this segment does not result in an active channel or cause neuronal degeneration (data not shown), as one would expect for a gateless channel. 
An alternative function for the $\mathrm{N}$-terminal segment could be to subserve as an anchor for the assembly of the signaling complex. It has been shown that the Drosophila TRP channel interacts with the PDZ (postsynaptic density-95/Discs large/zona occludens-1)-containing protein InaD, which acts as a scaffold to assemble singling components of phototransduction cascade (Shieh and Zhu, 1996; Chevesich et al., 1997; Tsunoda et al., 1997). Likewise, the adaptor protein Homer couples vertebrate TRPC1 (classical TRP) to the inositol triphosphate receptor (Yuan et al., 2003). In this model, the OCR-2 N-terminal segment contains targets for scaffold proteins, which in turn associate with multiple signaling components for activation of gene expression and for induction of behavioral responses. This model is particularly intriguing. Although the G36E substitution disrupts osmotic sensation mediated by ASH and downregulates tph-1 expression in $\mathrm{ADF}$, the underlying mechanism in these two functions appears to differ. Laser ablation experiments have indicated that ADF and ASH neurons sense different stimuli (Bargmann and Horvitz, 1991a). Unlike OCR-2/OSM-9-mediated osmotic sensation that requires the G $\alpha$-protein ODR-3 (Colbert et al., 1997; Tobin et al., 2002), tph-1 expression is unaffected in $o d r-3$ deletion mutants (Zhang et al., 2004). Consistent with the same notion, mouse and human TRPV2 substantially activate tph-1::gfp expression but cannot rescue the osmotic deficits in ocr-2 deletion mutants. Scaffold proteins have been proposed to act as "multivalent adaptors" (Tsunoda et al., 1997). For example, different PDZ domains in the PSD-95 (postsynaptic density-95) protein display preferences for distinct targets (Songyang et al., 1997). Therefore, the N-terminal segment may interact with a scaffold protein, which recruits different components to the OCR-2/OSM-9 channel in ADF and ASH neurons, thereby mediating neuron-specific functions. It is also possible that the $\mathrm{N}$ terminus contains multiple targets for different adaptor proteins or even directly interacts with neuron-specific signaling components, and that G36E alters the structures such that none of them can now bind. Isolation of ocr-2(yz5) suppressors will identify the components in the OCR-2/OSM-9 channel signaling and illuminate their function in specific sensory neurons. It will be interesting to determine whether the components acting in the ADF neurons are conserved in humans and whether there is a genetic linkage between these human homologs and 5-HT-related disorders.

\section{References}

Allessandri-Haber N, Yeh JJ, Boyd AE, Parada CA, Chen X, Reichling DB, Levine JD (2003) Hypotonicity induces TRPV4-mediated nociception in rat. Neuron 39:497-511.

Baldi P, Pollastri G (2003) The principled design of large-scale recursive neural network architectures-DAG-RNNs and the protein structure prediction problem. J Machine Learning Res 4:575-602.

Baldi P, Chauvin Y, Hunkapiller T, McClure MA (1994) Hidden Markov models of biological primary sequence information. Proc Natl Acad Sci USA 91:1059-1063.

Baldi P, Brunak S, Frasconi P, Soda G, Pollastri G (1999) Exploiting the past and the future in protein secondary structure prediction. Bioinformatics 15:937-946.

Bargmann CI, Horvitz HR (1991a) Chemosensory neurons with overlapping functions direct chemotaxis to multiple chemicals in C. elegans. Neuron 5:729-742.

Bargmann CI, Horvitz HR (1991b) Control of larval development by chemosensory neurons in Caenorhabditis elegans. Science 251:1243-1246.

Bargmann CI, Thomas JH, Horvitz HR (1990) Chemosensory cell function in the behavior and development of Caenorhabditis elegans. Cold Spring Harb Symp Quant Biol 55:529-538.

Bargmann CI, Hartwieg E, Horvitz HR (1993) Odorant-selective genes and neurons mediate olfaction in C. elegans. Cell 74:515-527.
Boels K, Glassmeier G, Herrmann D, Riedel IB, Hampe W, Kojima I, Schwarz JR, Schaller HC (2001) The neuropeptide head activator induces activation and translocation of the growth-factor-regulated $\mathrm{Ca}^{2+}$-permeable channel GRC. J Cell Sci 114:3599-3606.

Brenner S (1974) The genetics of Caenorhabditis elegans. Genetics 77:71-94. Caterina MJ, Rosen TA, Tominaga M, Brake AJ, Julius D (1999) capsaicinreceptor homologue with a high threshold for noxious heat. Nature 398:436-441.

Caterina MJ, Leffler A, Malmberg AB, Martin WJ, Trafton J, Petersen-Zeitz KR, Koltzenburg M, Basbaum AI, Julius D (2000) Impaired nociception and pain sensation in mice lacking the capsaicin receptor. Science 288:306-313.

Chevesich J, Kreuz AJ, Montell C (1997) Requirement for the PDZ domain protein, INAD, for localization of the TRP store-operated channel to a signaling complex. Neuron 18:95-105.

Clapham DE (2003) TRP channels as cellular sensors. Nature 426:517-524.

Colbert HA, Smith TL, Bargmann CI (1997) OSM-9, a novel protein with structural similarity to channels, is required for olfaction, mechanosensation, and olfactory adaptation in Caenorhabditis elegans. J Neurosci 17:8259-8269.

Culotti JG, Russell RL (1978) Osmotic avoidance defective mutants of the nematode Caenorhabditis elegans. Genetics 90:243-256.

de Bono M, Tobin DM, Davis MW, Avery L, Bargmann CI (2002) Social feeding in Caenorhabditis elegans is induced by neurons that detect aversive stimuli. Nature 419:899-903.

Delany NS, Hurle M, Facer P, Alnadaf T, Plumpton C, Kinghorn I, See CG, Costigan M, Anand P, Woolf CJ, Crowther D, Sanseau P, Tate SN (2001) Identification and characterization of a novel human vanilloid receptorlike protein, VRL-2. Physiol Genomics 4:165-174.

Denker SP, Barber DL (2002) Cell migration requires both ion translocation and cytoskeletal anchoring by the $\mathrm{Na}-\mathrm{H}$ exchanger NHE1. J Cell Biol 159:1087-1096.

Finney M, Ruvkun G (1990) The unc-86 gene product couples cell lineage and cell identity in C. elegans. Cell 63:895-905.

Guler AD, Lee H, Iida T, Shimizu I, Tominaga M, Caterina M (2002) Heatevoked activation of the ion channel, TRPV4. J Neurosci 22:6408-6414.

Gunthorpe MJ, Benham CD, Randall A, Davis JB (2002) The diversity in the vanilloid (TRPV) receptor family of ion channels. Trends Pharmacol Sci 23:183-191.

Harteneck C, Plant TD, Schultz G (2000) From worm to man: three subfamilies of TRP channels. Trends Neurosci 4:159-166.

Higgins D, Thompson J, Gibson T, Thompson JD, Higgins DG, Gibson TJ (1994) CLUSTAL W: improving the sensitivity of progressivemultiple sequence alignment through sequence weighting, position-specific gap penalties and weight matrix choice. Nucleic Acids Res 22:4673-4680.

Horvitz HR, Chalfie M, Trent C, Sulston JE, Evans PD (1982) Serotonin and octopamine in the nematode Caenorhabditis elegans. Science 216:1012-1014.

Jordt SE, Julius D (2002) Molecular basis for species-specific sensitivity to "hot" chili peppers. Cell 108:421-430.

Kanzaki M, Zhang YQ, Mashima H, Li L, Shibata H, Kojima I (1999) Translocation of a calcium-permeable cation channel induced by insulin-like growth factor-I. Nat Cell Biol 3:165-170.

Kaplan JM, Horvitz HR (1993) A dual mechanosensory and chemosensory neuron in Caenorhabditis elegans. Proc Natl Acad Sci USA 90:2227-2231.

Liedtke W, Friedman JM (2003) Abnormal osmotic regulation in trpv4-/mice. Proc Natl Acad Sci USA 100:13698-13703.

Liedtke W, Tobin DM, Bargmann CI, Friedman JM (2003) Mammalian TRPV4 (VR-OAC) directs behavioral responses to osmotic and mechanical stimuli in Caenorhabditis elegans. Proc Natl Acad Sci USA 100 [Suppl 2]:14531-14536.

McGuffin LJ, Bryson K, Jones DT (2000) The PSIPRED protein structure prediction server. Bioinformatics 16:404-405.

McIntire SL, Garriga G, White J, Jacobson D, Horvitz HR (1992) Genes necessary for directed axonal elongation or fasciculation in C. elegans. Neuron 8:307-322.

Mello CC, Kramer JM, Stinchcomb D, Ambros V (1991) Efficient gene transfer in C. elegans: extrachromosomal maintenance and integration of transforming sequences. EMBO J 10:3959-3970.

Mezey E, Toth ZE, Cortright DN, Arzubi MK, Krause JE, Elde R, Guo A, Blumberg PM, Szallasi A (2000) Distribution of mRNA for vanilloid receptor subtype 1 (VR1), and VR1-like immunoreactivity, in the central 
nervous system of the rat and human. Proc Natl Acad Sci USA 97:3655-3660.

Montell C, Birnbaumer L, Flockerzi V (2002) The TRP channels, a remarkably functional family. Cell 108:595-598.

Notredame C, Higgins D, Heringa J (2000) T-Coffee: a novel method for multiple sequence alignments. J Mol Biol 302:205-217.

Pollastri G, Baldi P, Fariselli P, Casadio R (2002a) Prediction of coordination number and relative solvent accessibility in proteins. Proteins 47:142-153.

Pollastri G, Przybylski D, Rost B, Baldi P (2002b) Improving the prediction of protein secondary structure in three and eight classes using recurrent neural networks and profiles. Proteins 47:228-235.

Raucher D, Stauffer T, Chen W, Shen K, Guo S, York JD, Sheetz MP, Meyer T (2000) Phosphatidylinositol 4,5-bisphosphate functions as a second messenger that regulates cytoskeleton-plasma membrane adhesion. Cell 100:221-228.

Roayaie K, Crump JG, Sagasti A, Bargmann CI (1998) The G alpha protein ODR-3 mediates olfactory and nociceptive function and controls cilium morphogenesis in C. elegans olfactory neurons. Neuron 20:55-67.

Schumacher MA, Moff I, Sudanagunta SP, Levine JD (2004) Molecular cloning of an $\mathrm{N}$-terminal splice variant of the capsaicin receptor. Loss of $\mathrm{N}$-terminal domain suggests functional divergence among capsaicin receptor subtypes. J Biol Chem 275:2756-2762.

Sengupta P, Chou JH, Bargmann CI (1996) odr-10 encodes a seven transmembrane domain olfactory receptor required for responses to the odorant diacetyl. Cell 84:899-909.

Shakir MA, Fukushige T, Yasuda H, Miwa J, Siddiqui SS (1993) C. elegans osm-3 gene mediating osmotic avoidance behaviour encodes a kinesinlike protein. NeuroReport 4:891-894.

Shieh BH, Zhu MY (1996) Regulation of the TRP Ca ${ }^{2+}$ channel by INAD in Drosophila photoreceptors. Neuron 16:991-998.

Smith GD, Gunthorpe MJ, Kelsell RE, Hayes PD, Reilly P, Facer P, Wright JE, Jerman JC, Walhin JP, Ooi L, Egerton J, Charles KJ, Smart D, Randall AD, Anand P, Davis JB (2002) TRPV3 is a temperature-sensitive vanilloid receptor-like protein. Nature 418:186-190.

Songyang Z, Fanning AS, Fu C, Xu J, Marfatia SM, Chishti AH, Crompton A, Chan AC, Anderson JM, Cantley LC (1997) Recognition of unique carboxyl-terminal motifs by distinct PDZ domains. Science 275:73-77.

Sze JY, Victor M, Loer C, Shi Y, Ruvkun G (2000) Food and metabolic signalling defects in a Caenorhabditis elegans serotonin-synthesis mutant. Nature 403:560-564.

Sze JY, Zhang S, Li J, Ruvkun G (2002) The C. elegans POU-domain transcription factor UNC-86 regulates the tph-1 tryptophan hydroxylase gene and neurite outgrowth in specific serotonergic neurons. Development 129:3901-3911.

Tobin D, Madsen D, Kahn-Kirby A, Peckol E, Moulder G, Barstead R, Maricq A, Bargmann C (2002) Combinatorial expression of TRPV channel proteins defines their sensory functions and subcellular localization in $C$. elegans neurons. Neuron 35:307-318.

Tominaga M, Caterina MJ, Malmberg AB, Rosen TA, Gilbert H, Skinner K, Raumann BE, Basbaum AI, Julius D (1998) The cloned capsaicin receptor integrates multiple pain-producing stimuli. Neuron 21:531-543.

Tsunoda S, Sierralta J, Sun Y, Bodner R, Suzuki E, Becker A, Socolich M, Zuker CS (1997) A multivalent PDZ-domain protein assembles signalling complexes in a G-protein-coupled cascade. Nature 388:243-249.

Vowels JJ, Thomas JH (1994) Multiple chemosensory defects in daf-11 and daf-21 mutants of Caenorhabditis elegans. Genetics 138:303-316.

Vriens J, Watanabe H, Janssens A, Droogmans G, Voets T, Nilius B (2004) Cell swelling, heat, and chemical agonists use distinct pathways for the activation of the cation channel TRPV4. Proc Natl Acad Sci USA 101:396-401.

Watanabe H, Davis JB, Smart D, Jerman JC, Smith GD, Hayes P, Vriens J, Cairns W, Wissenbach U, Prenen J, Flockerzi V, Droogmans G, Benham CD, Nilius B (2002) Activation of TRPV4 channels (hVRL-2/mTRP12) by phorbol derivatives. J Biol Chem 277:13569-13577.

White JG, Southgate E, Thomson JN, Brenner S (1986) The structure of the nervous system of the nematode C. elegans. Philos Trans R Soc Lond B Biol Sci 314:1-340.

Yellen G (2002) The voltage-gated potassium channels and their relatives. Nature 419:35-42.

Yuan JP, Kiselyov K, Shin DM, Chen J, Shcheynikov N, Kang SH, Dehoff MH, Schwarz MK, Seeburg PH, Muallem S, Worley PF (2003) Homer binds TRPC family channels and is required for gating of TRPC1 by IP3 receptors. Cell 114:777-789.

Zhang S, Sokolchik I, Blanco G, Sze JY (2004) Caenorhabditis elegans TRPV ion channel regulates $5 \mathrm{HT}$ biosynthesis in chemosensory neurons. Development 131:1629-1638. 